\title{
Atenção farmacêutica a saúde da pessoa idosa
}

\author{
Pharmaceutical care to the health of the elderly \\ Atención farmacéutica para la salud de los ancianos
}

Recebido: 01/12/2021 | Revisado: 07/12/2021 | Aceito: 08/12/2021 | Publicado: 13/12/2021

\author{
Juliana Santos Rocha Faria \\ ORCID: https://orcid.org/0000-0001-9228-2612 \\ Faculdade de Palmas, Brasil \\ E-mail: juhhsantos97@hotmail.com \\ Maykon Jhuly Martins de Paiva \\ ORCID: https://orcid.org/0000-0002-6228-4550 \\ Faculdade de Palmas, Brasil \\ E-mail: maykonjhulyfm@gmail.com
}

\begin{abstract}
Resumo
Este artigo tem como objetivo mostrar o papel fundamental do farmacêutico na saúde da pessoa idosa, uma classe tão essencial que muitas vezes fica desassistida, os idosos é a faixa etária que mais faz uso medicamentos no mundo, por isso há muito risco de se ter uma interação medicamentosa, já que a maioria deles tomam mais de 2 medicamentos por dia, o acompanhamento de um profissional habilitado é essencial para diminuirmos os riscos existentes, passar mais confiança para o tratamento que o idoso está fazendo, ensinar que as medicações devem ser tomadas no horário correto, e que não se pode parar um tratamento pela metade. Os idosos requerem muita atenção e zelo, pois em algumas situações eles nem sabem qual a finalidade dos inúmeros medicamentos que eles tomam, fazendo com que não terminem o tratamento corretamente, assim como os medicamentos de uso continuo, que não devem ser esquecidos de ser tomado. O farmacêutico é o profissional mais habilitado para esse suporte, devemos dar uma atenção maior para nossos idosos, já que muitas vezes o poder de entendimento deles é baixo, se deve ter um pouco mais de paciência e cautela, para fazer do tratamento medicamentoso algo que entre na rotina deles a ponto de não ser esquecido ou deixado de lado.
\end{abstract}

Palavras-chave: Idoso; Atenção farmacêutica; Medicamento.

\begin{abstract}
This article aims to show the fundamental role of the pharmacist in the health of the elderly, a class so essential that it is often unattended, the elderly is the age group that most uses medicines in the world so there is a right risk of having drug interaction, since most of them take more than 2 medications a day, the monitoring of a qualified professional is essential to reduce the existing risks, give more confidence to the treatment that the elderly is doing, teach that medications should be taken in the correct time, and that a treatment cannot be stopped in half. Elderly people require a lot of attention and care, as in some situations they do not even know the purpose of the numerous medications they take, causing them not finish the treatment correctly as well as continuous use medications, which should not be forgotten to be taken. The pharmacist is the most qualified professional for this support, we must pay great attention to our erderly, as their power of understanding is often low, a little more patience and caution must be exercised to make drug treatment something that get into their routine to the point where you won't be forgotten or left out.
\end{abstract}

Keywords: Old man; Pharmaceutical attention; Medicine.

\section{Resumen}

Este artículo tiene como objetivo mostrar el papel fundamental del farmacéutico en la salud de las personas mayores, una clase tan fundamental que muchas veces se encuentra desatendida, la tercera edad es el grupo de edad que más utiliza medicamentos en el mundo, por lo que existe un alto riesgo de tener interacción medicamentosa, dado que la mayoría toma más de 2 medicamentos al día, el seguimiento de un profesional calificado es fundamental para reducir los riesgos existentes, dar más confianza al tratamiento que está haciendo el anciano, enseñar que los medicamentos deben tomarse en la forma correcta tiempo, y que no puede detener un tratamiento a la mitad. Las personas mayores requieren mucha atención y cuidados, ya que en algunas situaciones ni siquiera conocen el propósito de los numerosos medicamentos que toman, provocando que no terminen correctamente el tratamiento, así como medicamentos de uso continuo, que no deben olvidarse. ser tomado. El farmacéutico es el profesional más calificado para este apoyo, debemos prestar mayor atención a nuestros mayores, ya que su poder de comprensión suele ser bajo, se debe ejercer un poco más de paciencia y cautela para hacer del tratamiento farmacológico algo que se meta en su rutina al punto donde no se le olvidará ni se le dejará fuera.

Palabras clave: Anciano; Atención Farmacéutica; Medicamento. 


\section{Introdução}

Vários problemas podem ocorrer com o uso de medicamentos por pessoas idosas, como o uso indevido do medicamento, como tomar o medicamento fora do horário ou esquecer de tomá-lo em determinado dia. sob o olhar atento, como os idosos não conseguem distinguir a dose normal, da dose próxima ao indicador tóxico, efeitos colaterais e interações medicamentosas também devem ser adicionados e monitorados, cerca de 1/3 dos adultos tomam cerca de cinco medicamentos diferentes por dia, o risco de interações medicamentosas é muito alto e precisa ser cuidadosamente considerado por um especialista qualificado para identificar essa combinação, explicar os efeitos colaterais contidos em cada medicamento, é importante aderir melhor ao tratamento, pois os idosos precisam saber como podem ver algo que mudará sua fisiologia.

O Brasil apresenta um perfil de mortalidade elevado, que passou das faixas etárias jovens para as avançadas, que precisam ser compostas por profissionais capacitados, a fim de tentar reverter essa tendência (Gordilho, et al 2000).

O atendimento médico é muito importante, farmácias e drogarias deveriam investir pesado nisso, mas muitos proprietários e gerentes não veem isso como muito importante, porque o farmacêutico tem muita responsabilidade pela gestão e administração do local de trabalho, ou seja, atendimento clinico é visto por muitos como custos desnecessários, mas será uma distribuição de saúde, mas há quem só queira dispensar dos os medicamentos e obter lucro.

Pacientes que apresentam mais de uma patologia, são submetidos a utilização de vários medicamentos, que muitas vezes podem trazer uma má resposta ao invés do resultado esperado ao seu tratamento. Desta forma temos a definição de polifarmácia como o uso subsequente de três ou mais medicamentos que são utilizados principalmente pela população da terceira idade. É muito rotineiro que os idosos utilizem a automedicação, por receio de buscar ajuda especializada e terem hábitos rotineiros limitados e errôneos, e com isso pode haver o surgimento de novas patologias induzidas pela automedicação, e piora nos quadros clínicos (Oliveira et al.,2021).

O farmacêutico clínico é um procedimento realizado na forma de atendimento ao paciente, aborda atitudes, valores, moral, competências, compromisso e cooperação na prevenção de doenças, promoção e recuperação da saúde, de forma integrada com a equipe de saúde.

É uma comunicação direta entre o farmacêutico e o consumidor, visando um tratamento médico favorável e a obtenção de resultados definidos e mensuráveis, visando a melhoria da qualidade de vida. Essa interação deve incluir também os conceitos de seus sujeitos, respeitando seus detalhes biopsicossociais, na perspectiva da integração das práticas de saúde (Ivama et al., 2002).

Com o aumento do número de idosos, é necessário focar nesses pacientes, grandes consumidores dos mais diversos tipos de medicamentos, alguns deles por recomendação médica e outros de uso com ou sem prescrição. $\mathrm{O}$ acompanhamento medicamentoso com o idoso é muito importante, para que ele siga o tratamento adequado, sem interrupção, e demonstre a importância da compreensão do uso da medicação.

No entanto, o atendimento médico aos idosos é importante, a fim de encontrar um tratamento eficaz e orientar os idosos a tomarem seus medicamentos diariamente e na hora certa, a pesquisa mostra que $50 \%$ dos compradores de medicamentos no mundo são idosos, e que, dentro desta lista, 1/3 deles tomam entre 3 a 5 medicamentos por dia, então atendimento de um farmacêutico habilitado para os idosos é necessário, porque há estudos que mostram que em $202530 \%$ da população mundial será mais velha, então é preciso começar agora esse cuidado especial, pois com o tempo teremos muitos idosos que tomam muitos remédios por dia, correm risco de toxicomania, interação medicamentosa e a auto medicação.

\section{Metodologia}

A metodologia do artigo é mostrar o quanto os idosos muitas vezes estão desassistidos, foi usados dados e informações de vários sites e publicações voltados para esse tipo de atenção farmacêutica, foi constatado que é a classe que 
mais faz uso de medicamentos, em torno de 5 fármacos por dia, o que pode causar diversos efeitos, tal como a interação medicamentosa, o alto índice de intoxicação, entre outros.

Os idosos necessitam de uma atenção especial, porque além dos fármacos que são prescritos, ainda há um alto índice de auto medicação, tanto por escolha deles, como aquela indicação que um "amigo" sempre tem para oferecer, porque ele acha que funcionou com ele, vai funcionar em todo mundo, e sabemos que não é assim, cada organismo pode reagir diferente a cada medicamento ingerido, assim como pode dar interação com outro fármaco que o idoso já faz uso, que sabemos que não são poucos, a automedicação é algo que me assusta muito, pois os índices são altíssimos, assim como se mostra na figura a seguir:

Figura 1. Pesquisa auto medicação no Brasil (ICTQ 2018).

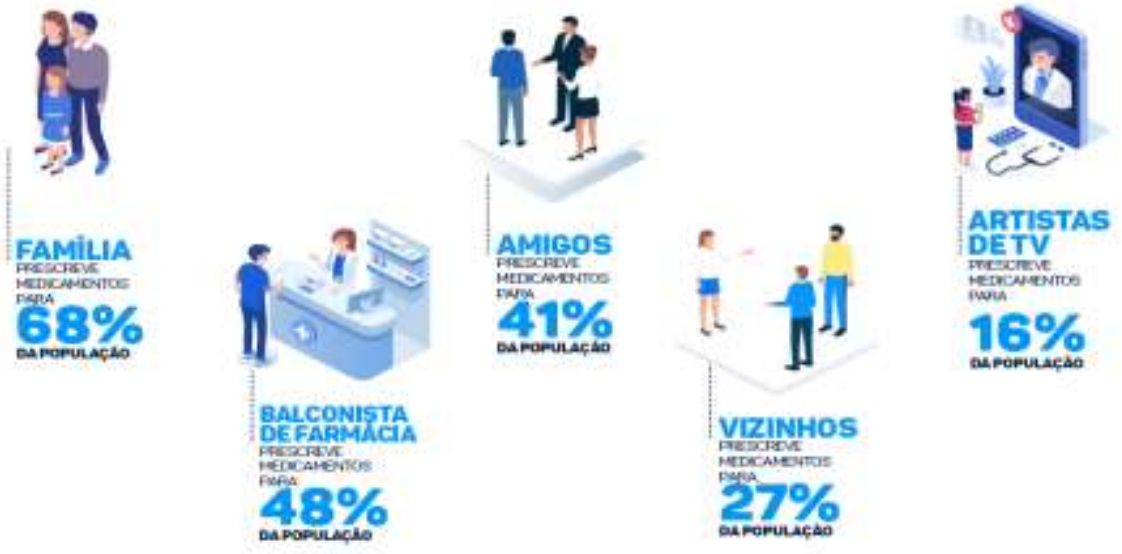

Fonte: ICTQ Pesquisa.

Com isso se vê que ate as propagandas de TV influenciam a automedicação, e o índice é maior em idosos, que muitas vezes pela fala de conhecimento julga tudo que se passa na propaganda ser correto, então o acompanhamento farmacêutico para essa classe é fundamental, nossos idosos precisam ser assistidos de perto, precisa serem orientados ao correto uso de medicamentos, e entender que a auto medicação é uma atitude errada e que precisa ser repensada.

\section{Resultados e Discussão}

A população idosa é o grupo de pessoas que mais cresce no Brasil, eles são os maiores consumidores de medicamentos no mundo, devido a esse grande consumo, o Brasil ocupa a sexta posição do mercado mundial como um dos maiores consumidores de medicamentos (Oliveira \& Corradi, 2018).

Assistência Farmacêutica envolve um consenso entre um paciente, que aceita a autorização de um farmacêutico, e um farmacêutico que confirma o entusiasmo e dedicação do paciente. A adesão inadequada ao tratamento adequado compromete os esforços do sistema de saúde, cria complicações e reduz a qualidade de vida das pessoas que utilizam esse tratamento (International Council of Nursis, 2014).

A automedicação pode levar a uma série de efeitos colaterais, como resistência à infecção, dependência e aumento do risco de certos tipos de câncer. Segundo Relvas (20013), os riscos de interações medicamentosas em idosos podem ser reduzidos corrigindo alguns erros de automedicação, na dosagem, adesão à medicação, interrupção ou continuidade necessária.

Essa atenção dispensada ao farmacêutico visa orientar, esclarecer e acompanhar a pessoa, que busca administrar de forma eficaz e contínua o tratamento medicamentoso e diminuir os efeitos colaterais graves, pois os idosos estão na categoria da especialidade, pois uma entre tantas pessoas está em risco para o medicamento problemas. 
Com orientação e cuidados adequados, os idosos podem melhorar sua qualidade de vida e, com isso, aumentar sua expectativa de vida. Assim, o farmacêutico deve ser um especialista acessível para ouvir, tirar dúvidas, realizar a adesão à farmacoterapia, quando necessário e ser corajoso.

Essas ações são importantes para reduzir as reações adversas e aumentar o tratamento médico atual. No processo de envelhecimento, o idoso realiza mudanças biológicas, físicas, cognitivas, patológicas e sociais, necessitando de cuidados especiais. No entanto, os dados de idade não implicam que uma pessoa idosa seja uma pessoa com doença terminal, mas que essas mudanças podem variar de acordo com um estilo de vida ativo e saudável.

É importante que os profissionais de saúde conheçam os fatores determinantes desse processo, entendam sua complexidade e trabalhem na promoção da saúde desses idosos. Portanto, uma das formas mais importantes de garantir a independência dos idosos, bem como um envelhecimento saudável, é por meio de atividades educativas.

A educação em saúde visa, então, promover o desenvolvimento do conhecimento e, assim, melhorar a saúde e o bemestar das pessoas que participam desse processo. A realização de atividades que envolvam atividades educativas com adultos torna-se uma forma de ampliar o debate sobre essas questões, bem como possibilitar a formação de profissionais aptos a cuidar da doença (Larissa, 2016).

Os cuidados com a medicação também devem ter como objetivo promover o uso consciente de medicamentos. Nesse sentido, busca informar aos idosos sobre os problemas relacionados ao uso de drogas, sem discriminação.

O farmacêutico deve apoiar sempre o paciente e não o comércio descontrolado de drogas, o que tem aumentado o uso de medicamentos não prescritos por um profissional de saúde, pois as drogas ilícitas estão entre as mais utilizadas (Fernandes \& Cembranelli, 2015).

\subsection{Contribuição da indústria farmacêutica promova a saúde}

Dentro desta nova indústria farmacêutica, onde a ansiedade está em causa do paciente torna-se um pilar de ação, o farmacêutico tem um papel importante, somando seus esforços aos de outros profissionais, saúde e promoção do bem estar da comunidade. Autores James e Rovers (2003) identificaram quatro categorias de programas possíveis e feito por farmacêuticos para melhorar a saúde pública:

1. Monitoramento, ensino e atuação ao paciente;

2. Avaliação de risco;

3. Prevenção de saúde;

4. Promoção da saúde e monitoramento de doenças.

E segundo os autores acima, a promoção da saúde pode ser feita por meio de três domínios que dão suporte aos serviços de prestação de serviços de proteção clínica;

\subsection{Monitoramento e publicação de saúde pública e promover o uso sustentável das comunidades.}

A abordagem da James e Rovers (2003) está em conformidade com os tópicos listados na lista da OMS24 em relação à possível ação promoção de saúde. Nossa tradução literal de técnicas protegidas em todo o mundo, é possível dizer que o farmacêutico (de uma drogaria, ou de uma farmácia hospitalar privada e ou unidades de saúde do paciente) podem operar em três pontos básicos: redesenhar o serviço de farmácia, melhorar as competências a comunidade também incentivar os indivíduos a ação pública e que são prestados população.

\subsection{Aconselhamento da farmácia}

Estudos realizados em farmácias sobre o manejo da diarreia por meio da terapia de reidratação oral concluíram que esses centros podem ser postos de saúde se utilizados de maneira adequada. Isso pode refletir a importância do papel na saúde 
pública, diferente do processo que até agora foi observado. Há trinta anos, farmácias e drogarias particulares recebiam pedidas e entregam produtos compatíveis aos usuários do serviço, quase sempre sem nenhum tipo de orientação sobre seu uso. A gestão de fórmulas não escapou a esta lei.

No que diz respeito aos serviços públicos, governos e líderes discutem a questão da entrega de medicamentos e estratégias financeiras, mas poucos percebem que os medicamentos são simplesmente uma ferramenta para a prestação de serviços e muitas vezes não se preocupam com a organização do serviço. A maioria das farmácias em unidades básicas de saúde funciona porque um paramédico ou profissional médico menos experiente atua na distribuição (auxiliares de enfermagem, auxiliares administrativos, auxiliares da própria farmácia, entre outros).

Entre as muitas necessidades e requisitos, os serviços de farmácia não são considerados uma prioridade no orçamento de recursos. Talvez seu valor não seja claro para muitos gerentes. Isso pode ser verificado a partir da formação dos recursos humanos em que estão inseridos, embora sejam necessárias pesquisas sobre o tema. Na construção de unidades de saúde, as farmácias costumam se distribuir em uma área pequena, e geralmente não há condições mínimas.

É necessário armazenar o medicamento de maneira adequada. Ainda é possível encontrar farmácias com barreiras que separam os usuários dos profissionais que os prestam. Além disso, há falta de pessoal qualificado. Portanto, o serviço não tem condições suficientes para realizar sua função e relacionamento personalizado.

É importante lembrar que faltam serviços de farmácia adequados para garantir o uso racional dos medicamentos em articulação com outros serviços. Reposicionamento de serviços de farmácia. É preciso estar atento ao uso razoável dos medicamentos, para que o paciente receba a medicação com a indicação certa, as dosagens certas, a via de administração e o horário; que não há contradição; que as chances de uma disputa menor surgir são mínimas; que o compartilhamento é direto e aderente ao tratamento.

Os serviços médicos de atenção primária contribuem para reduzir a hospitalização ou o tempo de internação hospitalar, cuidados para pacientes com doenças crônicas, praticar a educação para a saúde, bem como intervenções médicas menos dispendiosas. Dentro desse conceito, o serviço de farmácia deve assumir um papel coerente no serviço médico de atenção à saúde. Um paciente fora do consultório com uma receita terá uma solução maior para seus problemas se conseguirem receber tratamento prescrito e se o médico atende à racionalidade médica.

Mas isso não é tudo. Também é necessário verificar se há coisas que podem interferir no seu tratamento, como: hábitos alimentares, tabagismo, histórico de alergias, uso de certos medicamentos ou drogas, outras doenças, ou mesmo falta de adesão. Este teste, que é possível intervenções voltadas a cuidados médicos eficazes podem ser alcançadas por meio da implementação de cuidados farmacológicos.

O farmacêutico, via de regra, é o último profissional de saúde que se comunica diretamente com o paciente após uma decisão médica pelo tratamento. Dessa forma, você se torna responsável por sua própria qualidade de vida. Tanto o usuário quanto o profissional devem estar totalmente identificados onde estão e, portanto, os conceitos de pessoa, responsabilidade, respeito, verdade, consciência, independência, justiça, etc. eles devem ser incorporados a fim de exercer o profissionalismo.

Acabou-se a personalização do serviço de farmácia de todos esses itens e também cobre questões relacionadas à natureza do serviço. Espaço adequado é necessário para trazer prosperidade e autoconfiança. O farmacêutico poderá auxiliá-lo na sala reservada para este fim, garantindo a privacidade. Portanto, a formação de intervenções farmacológicas no sistema farmacêutico é um importante meio de promoção da saúde.

\subsection{Estratégias para o cuidado farmacêutico em idosos}

Faber et. al. (2017), em estudo realizado com idosos em instituições, constatou que permanecer mais tempo não significa que estejam em forma, saudáveis. Na prática, o envelhecimento está associado a um maior número de doenças 
crônicas que causam diminuição da atividade e aumento da dependência dos idosos. Portanto, a polimedicação é um fator importante de risco para complicações relacionadas aos medicamentos e representa um desafio para lidar com o rápido processo de crescimento em idosos, que é um fenômeno mundial.

Infelizmente, os medicamentos podem interagir com a retenção de energia, se prescritos e usados corretamente, ou podem ser muito prejudiciais, se houver recusa em prescrevê-los e usá-los. No caso dos idosos, a preocupação é ainda maior, pois os medicamentos prescritos devem ser avaliados quanto à gravidade de seu risco para não comprometer a saúde desses indivíduos (Carvalho, et al, 2012).

Com a idade, os órgãos perdem grande parte de sua capacidade funcional, incluindo o coração, o fígado e os rins. Junto com isso, a erosão da homeostase contribui para aumentar os riscos de medicamentos prescritos em idosos. Portanto, o atendimento farmacêutico ao idoso é uma importante ferramenta utilizada pelos farmacêuticos para promover o uso racional de medicamentos e conscientizar a população sobre a importância dessa prática (Fernandes \& Cembranelli, 2015).

O cuidado químico com o idoso sempre foi de suma importância, atualmente, é tão importante como parte das estratégias de cuidado à saúde, pois está diretamente ligado à promoção, cuidado e restauração do bem-estar. Com esse cuidado, é possível evitar a recorrência de doenças, principalmente com o uso adequado de medicamentos. O especialista supervisiona o paciente, garante que o medicamento prescrito tem o efeito desejado e alerta para possíveis interações, reações adversas e intoxicações (Lima et al., 2016).

Devido a vários problemas médicos, o tratamento dos idosos está se tornando cada vez mais complexo. Por isso, o farmacêutico tem sido chamado a participar de diversos grupos de atenção ao idoso, por ser um profissional de saúde com conhecimento de todos os aspectos e propriedades do medicamento. Como resultado, contém informações confidenciais, que devem ser compartilhadas com os indivíduos para garantir o uso adequado dos medicamentos e proteger as interações medicamentosas, para garantir a eficácia do tratamento e para reduzir o risco de lesões aos pacientes (Pinto; Castro; Reis, 2013).

Aliberti et. al. (2016) propuseram um modelo de atenção ao idoso com doenças crônico-degenerativas em que o farmacêutico desempenha um papel fundamental nas práticas educativas em relação ao tratamento, enfatizando a adesão ao tratamento e a resolução de problemas. Para atuar no cuidado ao idoso, o farmacêutico necessita de empatia, praticidade, flexibilidade, habilidade de comunicação, habilidade para lidar com situações complexas e facilidade de comunicação com os demais membros da equipe. Suas funções devem ser sempre pautadas por princípios éticos, de respeito a toda a sua diversidade, buscando proteção, promoção e reabilitação. Habilidades básicas para cuidar de idosos incluem informações sobre: farmacologia médica e farmacoterapia usada em idosos, parâmetros farmacocinéticos e possíveis mudanças na velhice, interpretação de testes, fisiopatologia, políticas de policiamento comunitário e escalas para testes geriátricos completos (Brasil, 2020).

O farmacêutico deve prestar atenção especial a coisas como possíveis medicamentos, interações medicamentosas, doses menores ou maiores necessárias, reações adversas, uso impróprio, automedicação e reações adversas de doses. Isso é feito por meio de anamnese e teste das medicações utilizadas pelo paciente. $\mathrm{O}$ farmacêutico fornece informações sobre as doenças e medicamentos que lhe são administrados e avisa o autor das complicações medicamentosas que identificou, buscando melhorar a adesão ao tratamento e reduzir os riscos associados ao cuidado durante o acompanhamento farmacoterapêutico. (Quinalha \& Correr, 2011)

Em seu estudo, Guimarães et. al. (2012) identificaram um número significativo de medicamentos de venda livre para idosos. O uso de medicamentos prescritos revela o desconhecimento de alguns médicos quanto ao fornecimento de medicamentos inadequados a esse grupo de pessoas. Isso pode ter efeitos colaterais graves para a clínica e aumentar o custo do sistema de saúde por meio de seu reparo. 
Neves et. al. (2013) também encontraram altas doses de medicamentos adversos em idosos, pois 21,6\% dos pacientes com polimedicina em seu estudo usavam pelo menos um medicamento considerado inseguro para a idade. Nesse contexto, Pinto, Castro e Reis (2013) destacam a importância de o farmacêutico analisar a farmacoterapia após a coleta de dados, levando em consideração referência, eficácia, segurança, posologia, características biofarmacêuticas em relação ao manejo da patologia, estabilidade estrutural, alinhamento físico-químico de medicamentos parenterais e interações medicamentosas. Se, após essa análise, o especialista julgar necessário, devem ser propostas intervenções médicas, com o objetivo de contribuir para a eficácia e segurança do tratamento medicamentoso, as orientações necessárias são sempre dadas aos pacientes e familiares.

O acompanhamento de pacientes idosos com farmacoterapia permite prevenir possíveis erros de prescrição e complicações relacionadas aos medicamentos. A atuação do farmacêutico também pode se estender à residência do paciente, para orientar cuidadores e familiares sobre aspectos relacionados ao armazenamento e gerenciamento de medicamentos, para desenvolver ferramentas que possam facilitar o tratamento e promover o uso eficaz de medicamentos (Olmedilha; Cappelaro, 2013).

É certo que a atuação do farmacêutico é determinante na qualidade de vida do idoso. Detalhes sobre medicamentos prescritos, reações adversas, interações medicamentosas e outros problemas relacionados aos medicamentos, bem como informações sobre seu uso adequado, fazem do especialista um importante agente na farmácia médica dessa faixa etária. As ações realizadas pelo farmacêutico durante a consulta incluem reconciliação de medicamentos, teste de adesão ao tratamento e monitoramento de reações adversas e interações medicamentosas (Brasil, 2020).

A literatura comprova que o atendimento farmacêutico permite a identificação, resolução e prevenção de problemas relacionados ao uso de drogas em idosos. Ao receber esse cuidado, o paciente tem uma melhor resposta ao tratamento de suas enfermidades. Portanto, é muito importante que a assistência médica seja incluída nos serviços de saúde (Fontana, 2015; Silva; Aragão; Sabino, 2016).

\section{Conclusão}

O número de idosos na sociedade brasileira está crescendo rapidamente, o envelhecimento é acompanhado por mudanças no perfil da população e, em decorrência, do crescente uso de medicamentos por esse grupo de pessoas, tornando-os mais vulneráveis às complicações relacionadas às drogas. . .

Por meio deste estudo, ficou comprovada a importância da comunicação entre o farmacêutico e o paciente, principalmente no papel do farmacêutico no acompanhamento da farmacoterapêutica do idoso. As drogas são abreviadas como um fator importante na aquisição e garantia da qualidade da saúde, resumidas nos primeiros elementos que constituem ferramentas poderosas para aliviar o sofrimento humano. Produzem tratamento, prolongam a vida e retardam o aparecimento de problemas relacionados à doença, promovendo a convivência com a doença. Por outro lado, podem aumentar o custo dos cuidados de saúde se forem mal utilizados ou levar ao consumo de drogas. Cabe ao farmacêutico, por meio de assistência médica, garantir que o tratamento médico razoável seja seguro para o paciente, a fim de proporcionar uma vida melhor para o idoso, reduzir a automedicação e o número de interações medicamentosas.

\section{Referências}

Aliberti, M. J. et al (2016) The Geriatric Day Hospital: dados preliminares sobre um modelo inovador de atenção ao idoso em risco de hospitalização no Brasil. Journal of the American Geriatrics Society, 64(10), 2149-2153.

Brasil. (2020) Conselho Regional de Farmácia do Estado de São Paulo. Departamento de Apoio Técnico e Educação Permanente. Grupo Técnico de Trabalho de Cuidado Farmacêutico ao Idoso. Cuidado Farmacêutico ao Idoso. São Paulo: Conselho Regional de Farmácia do Estado de São Paulo.

Carvalho, M. F. C. et al. (2012) Polifarmácia entre idosos do Município de São Paulo - Estudo SABE. Revista Brasileira de Epidemiologia, $15(4)$, 817- 27.

Coradi, A. E. P. (2012). A importância do farmacêutico no ciclo da Assistência Farmacêutica. Arquivos Brasileiros de Ciências da Saúde, 37(2), 62-64. http://files.bvs.br/upload/S/1983-2451/2012/v37n2/a3051. 
Research, Society and Development, v. 10, n. 16, e488101624224, 2021 (CC BY 4.0) | ISSN 2525-3409 | DOI: http://dx.doi.org/10.33448/rsd-v10i16.24224

Faber, L. M. \& Scheicher, M. E Soares, E. (2017). Depressão, declínio cognitivo e polimedicação em idosos institucionalizados. Revista Kairós Gerontologia, 20(2), 195-210.

Fernandes, W. S. \& Cembranelli, J. C. (2015). Automedicação e o uso irracional de medicamentos: o papel do profissional farmacêutico no combate a essas práticas. Revista Univap, 21(37), 5-12.

Fontana, R. M. et al. (2015). Atenção farmacêutica a pacientes hipertensos e/ou diabéticos usuários de farmácias públicas do município de lajeado-rs. Revista Destaques Acadêmicos, 7(3).

Gordilho, A et al. (2010) Desafios a serem enfrentados no terceiro milênio pelo setor saúde na atenção integral ao idoso. Universidade aberta da terceira idade UERJ.

Guimarães, V. G. et al. (2012). Perfil Farmacoterapêutico de um Grupo de Idosos assistidos por um programa de Atenção Farmacêutica na Farmácia Popular do Brasil no município de Aracaju-SE. Revista Ciências Farmacêuticas Básica Aplicada, 33(2), 307-312.

International Council of Nursis. (2014). Conselho Internacional de Enfermeiras.

Ivama, A. M., Noblat, L., Castro, M. S., Jaramillo N. M., \& Rech, N. (2002). Consenso Brasileiro de Atenção Farmacêutica: proposta. Brasília; OPAS.

James J. A, \& Rovers J. P. (2003) Wellness and health promotion. In: Rovers JP, et al. Um guia prático para assistência farmacêutica. p.183-200.

Larissa, S., Oliveira, R., Quirino, C. E., Nogueira, K. B., \& Bezerra, M. L. (2016). Serviço de atendimento farmacêutico ao idoso: relato de experiência de educação em saúde. Saúde Santa Maria.

Lima, T. A. M. et al. (2016). Acompanhamento farmacoterapêutico em idosos. Revista Arquivos de Ciências da Saúde, 23(1), 52-57.

Oldemilha, R. S \& Cappelaro, A. M. S.(2013). O papel do farmacêutico na Atenção Domiciliar. Revista de Pesquisa e Inovação Farmacêutica. 5(1), Universidade Anhanguera de São Paulo.

Oliveira, P. C. et al. (2021) Prevalência efatores associados à polifarmácia em idosos atendidos na atenção primária à saúde em Belo Horizonte-MG, Brasil. Ciência \& Saúde Coletiva. https://www.cienciaesaudecoletiva.com.br/artigos/prevalencia-e-fatores-associados-a-polifarmacia-em-idosos-atendidos-naatencao-primaria-a-saude-em-belo-horizonte-mg/17294?id=17294\&id=17294.

Pesquisa auto medicação no Brasil (ICTQ 2018). Figura 1.

Pinto, I. V. L \& Castro, M. dos S. \& Reis, A. M. M. (2013). Descrição da atuação do farmacêutico em equipe multiprofissional com ênfase no cuidado ao idoso hospitalizado. Rev. Bras. Geriatr. Gerontol., 16(4), 747-758.

Quinalha, J. V.; Correr, J. C. (2011). Instrumentos para avaliação da farmacoterapia do idoso: uma revisão. Revista Brasileira de Geriatria e Gerontologia, 13(3), 487-499.

Relvas, R.P. (2013). Em busca de uma assistência adequada á saúde do idoso. Cad Saúde pública.

Silva, L. G. A. \& Aragão, C. C. V. \& Sabino, W. (2016). Pressão arterial e atenção farmacêutica: o cuidado faz a diferença. Revista de Atenção à Saúde, 14(47), 12-18. 